\title{
Endoscopic ultrasound-guided fine needle biopsy of pancreatic metastasis from Merkel cell carcinoma
}

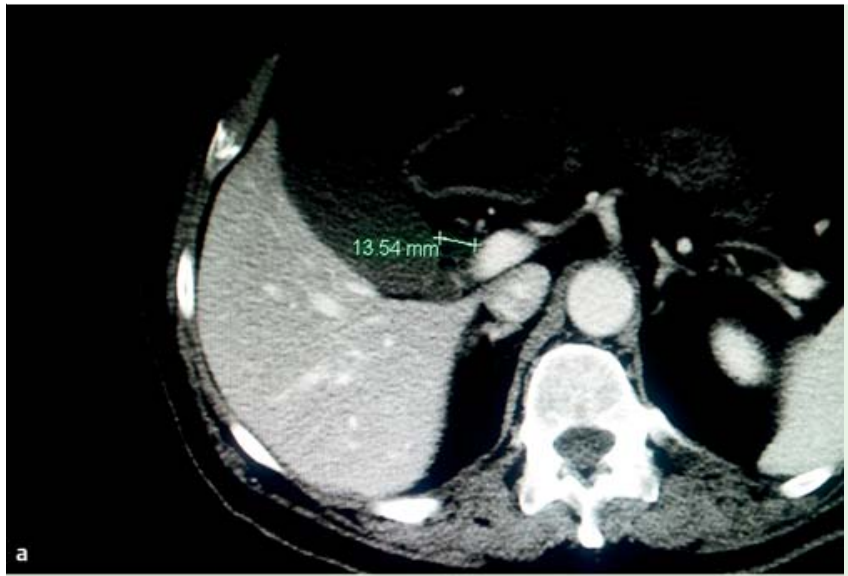

Fig. 1 Computed tomography (CT) scan images of the abdomen showing: a a 3-cm solid, irregular mass in the pancreatic head without vascular involvement; $\mathbf{b}$ the dilated common bile duct $(1.4 \mathrm{~cm})$ upstream of the lesion, a picture suggestive of a resectable pancreatic adenocarcinoma.
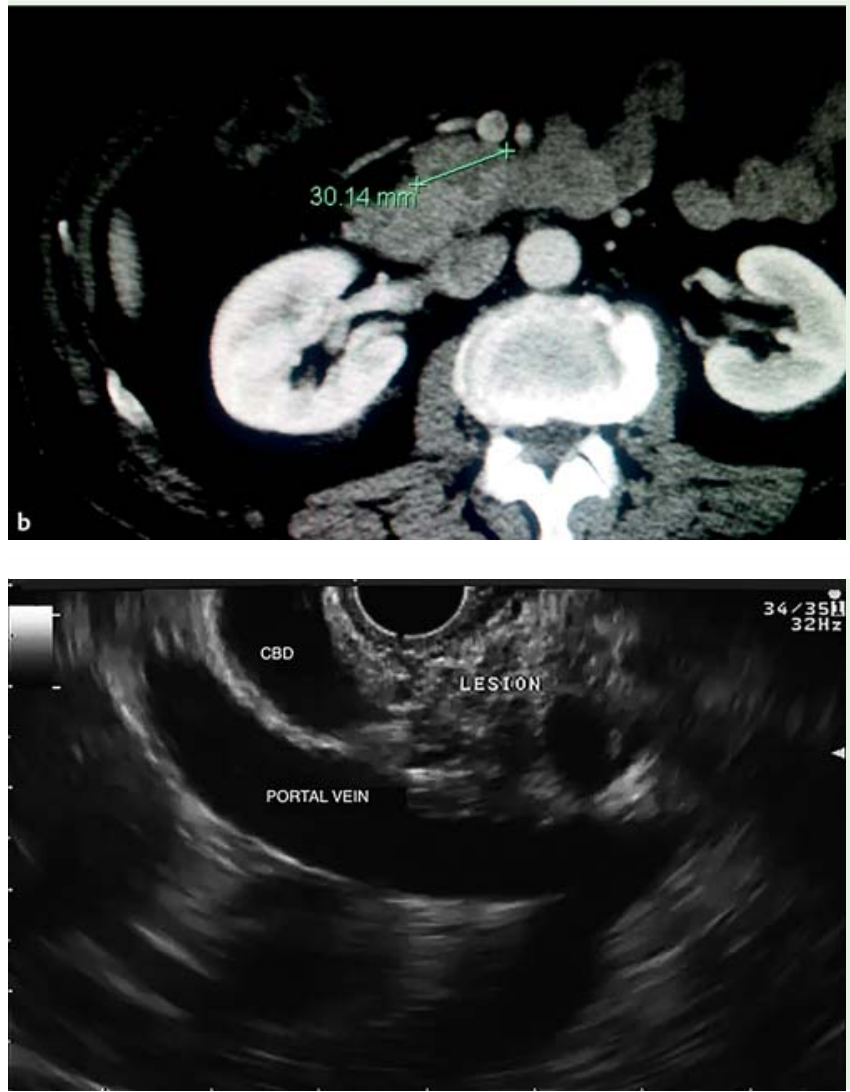

Fig. 2 Endoscopic ultrasound image of the pancreatic head showing a hypoechoic lesion with anechoic gaps within it that was causing stenosis of the terminal common bile duct (CBD) and dilatation upstream of the lesion. This lesion, with irregular margins, appeared to be infiltrating the portal confluence.

Merkel cell carcinoma (MCC) is a rare and aggressive cutaneous neuroendocrine carcinoma. The incidence rate is approximately $0.3-0.6 / 100000$ per year $[1,2]$. At initial presentation most patients with MCC (70\%-80\%) have localized disease, and only a few (1\%-4\%) have distant metastases [3]. Moreover, MCC rarely metastasizes to the pancreas, therefore this represents a challenge for the differential diagnosis of pancreatic masses [4].
A 73-year-old man reported epigastric pain and vomiting. The patient's history included a diagnosis of an MCC, which had been removed from his left elbow 7 months before the onset of his upper gastrointestinal symptoms. His laboratory findings were unremarkable. An abdominal computed tomography (CT) scan showed a lesion infiltrating the common bile duct in the pancreatic head, without vascular involvement, which was consid-

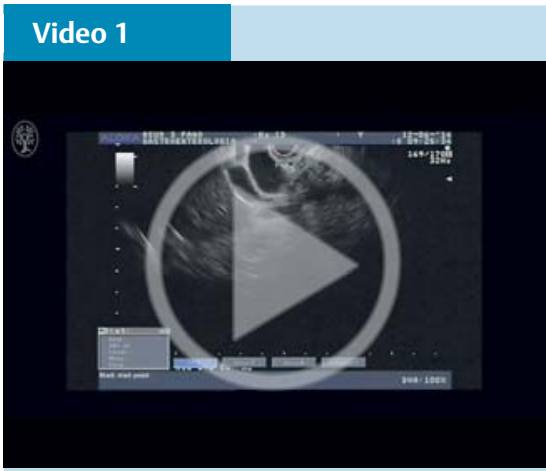

Endoscopic ultrasound of the pancreatic head showed a 3-cm hypoechoic, non-homogeneous lesion that appeared to be infiltrating the portal confluence. The common bile duct upstream of the mass appeared dilated.

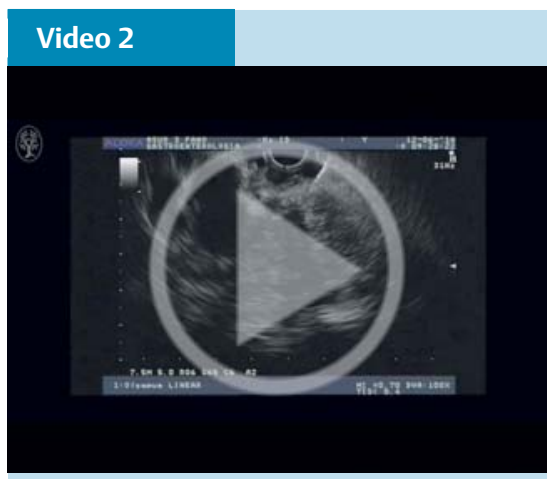

Three needle passes, using a "fanning" technique followed by slow withdrawal of the stylet, were performed with a 22-gauge ProCore needle.

ered to be a possibly resectable adenocarcinoma ( $\bullet$ Fig. 1 ).

The patient underwent an endoscopic ultrasound (EUS), which confirmed a 3-cm hypoechoic, heterogeneous, irregular mass with evidence of invasion of the portal confluence ( $\bullet$ Fig. 2; $\bullet$ Video 1 ). Three needle passes were performed with a 22-gauge ProCore needle (Cook Medical, Winston-Salem, North Carolina, USA) using a "fanning" technique followed by slow withdrawal of the stylet ( $\bullet$ Video 2 ). Cytohistological evaluation of the samples revealed small blue, round-to-oval cells with stippled chromatin ( Fig.3a). The cells were positive for CK20, sinaptophysin, and chromogranin, and had a Ki-67 index of $>60 \%$, suggestive of pancreatic metastasis from MMC ( $\bullet$ Fig. 3 b). 


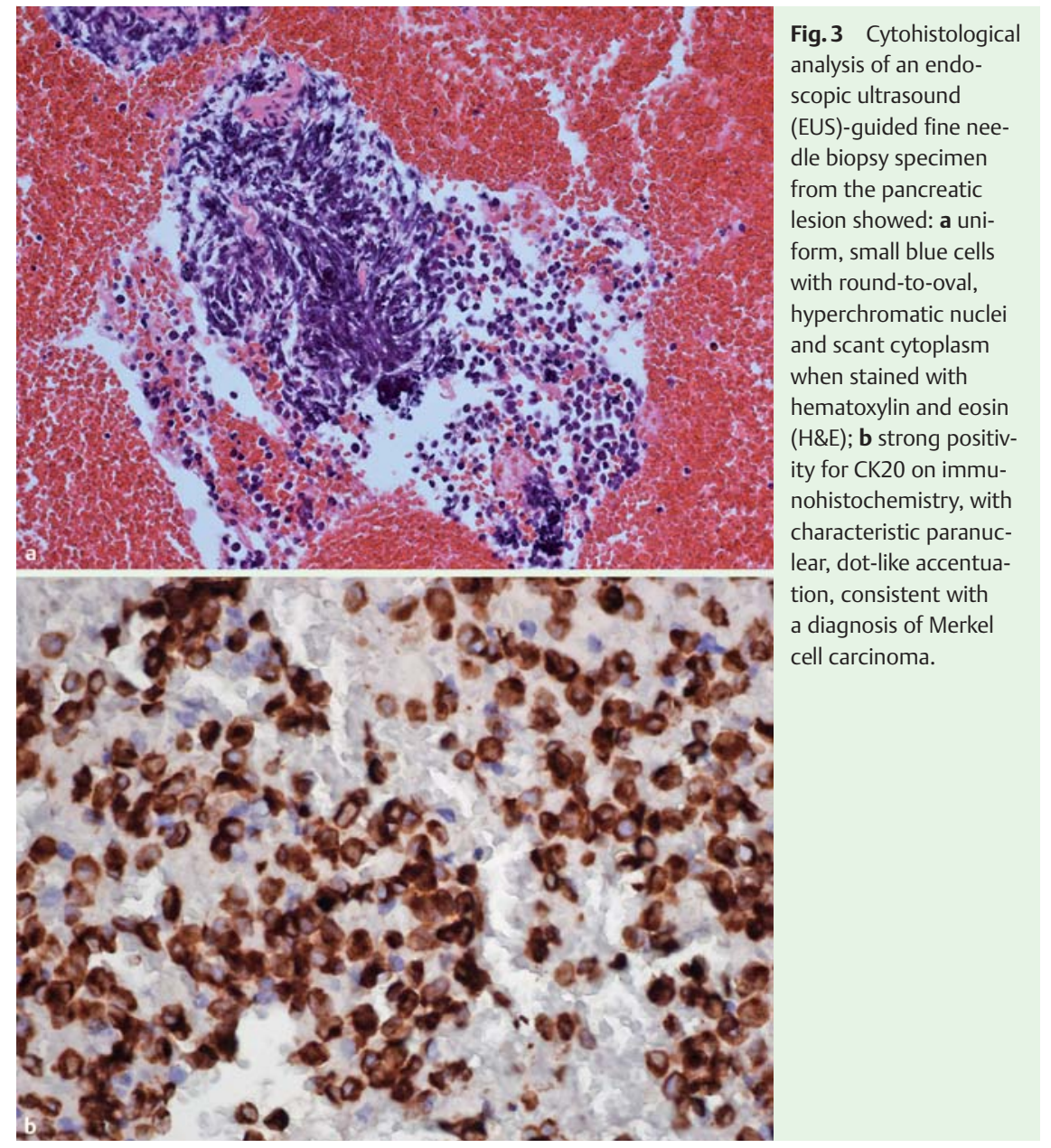

In this specific case, the EUS features of the pancreatic metastasis from MMC mimicked a classic adenocarcinoma. Moreover, this neoplasm showed few specific cytologic features as the same small blue, round-to-oval cells can also be seen in lymphoma or small cell carcinoma [5]. Given that CK20 is a pathognomonic marker of MCC [4,5], obtaining an adequate tissue sample for immunohistochemical evaluation with the use of an

\section{Antonella Maimone', Maria Luisa Bianchi ${ }^{1}$, Paola Lorenzini ${ }^{2}$, Annalisa de Leone ${ }^{1}$, Luca De Luca ${ }^{1}$}

${ }^{1}$ Gastroenterology and Digestive Endoscopy Unit, A.O. "Ospedali Riuniti Marche Nord”, Pesaro, Italy

${ }^{2}$ Department of Pathological Anatomy, A.O. "Ospedali Riuniti Marche Nord", Pesaro, Italy

\section{References}

1 Becker JC. Merkel cell carcinoma. Ann Oncol 2010; 21: $81-85$

2 Oulette JR, Woodyard L, Toth $L$ et al. Merkel cell carcinoma metastatic to the head of the pancreas. JOP 2004; 5: 92-96

3 Goessling W, McKee PH, Mayer RJ. Merkel Cell Carcinoma. J Clin Oncol 2002; 20: 588 598

4 Ghouri YA, Krishna SG, Kundu UR et al. A case series and literature review of Merkel cell carcinoma metastasizing to pancreas. Dig Dis Sci 2015; 60: 1805-1812

5 Bechert CJ, Schnadig VJ, Nawgiri RS. The Merkel cell carcinoma challenge: a review from the fine needle aspiration service. Cancer Cytopathol 2013; 121: 179-188

\section{Bibliography}

DOI http://dx.doi.org/

10.1055/s-0042-108569

Endoscopy 2016; 48: E199-E200

(c) Georg Thieme Verlag KG

Stuttgart · New York

ISSN 0013-726X

\section{Corresponding author}

\section{Luca De Luca, MD}

Gastroenterology and Digestive Endoscopy Unit A.O. "Ospedali Riuniti Marche Nord"

Piazzale Cinelli 1

61121, Pesaro

Italy

Fax: +39-072-1362285

lucadeluca1210@gmail.com 